\title{
Heisenberg spin textures on a cylinder with topological defects
}

\author{
L.A.N. de Paula* \\ Department of Mechanics and Material Physics, São Paulo University \\ Rua do Matão Travessa R 187, Butantã, 05508-090, São Paulo, SP, Brazil.
}

(Received on 9 September, 2009)

\begin{abstract}
The present work aims to study equilibrium configurations of spins on a cylinder with topological defects such as screw dislocation and deficit angle. By making use of elliptic-f expansion method, which in turn utilizes the Jacobi elliptic functions, we obtain exact solutions of the nonlinear sigma model in this geometry. We have significant changes in the qualitative behavior of the solutions due to the presence of the parameter $k$ of screw dislocation. In particular, the behavior of soliton-like solutions, characteristic of a cylinder without dislocation, was not found in the model here proposed.
\end{abstract}

Keywords: Heisenberg's spins - nanomagnetism

The nonlinear sigma model is the continuous limit of Heisenberg's hamiltonian for spins and has gained interest recently. It allows the study of equilibrium configurations of spins on non-trivial geometries such as cylinders, tori, ellipsoids, surfaces with negative curvature and so on [1-4]. The study of the stability of spin configurations and geometric frustration on different kinds of geometry are the two effects most explored with this model [5-8]. Defects such as punctures and impurities in the structure have also played an important role related to spin topological textures. In particular, we have introduced topological defects in an ideal cylinder such as screw dislocation. This defect is obtained by producing a longitudinal displacement in the structure, distorting the lattice helically. In this way we can study how the spin textures are modified in relation to the simple cylinder without these defects.

Connections with fundamental areas and important technological applications can also be made: the "similarity" between the nonlinear sigma model in 2D and Yang-Mills in $(3+1) \mathrm{D}$ [9]; out-of-plane vortex (its core is similar to the central region of a soliton of spins). These out-of-plane vortex are important in several mechanisms of magnetic logic (MRAM), ultra-precise magnetic sensors, magnetic recording, etc. [10, 11]

The Heisenberg model takes into account only the interaction of each spin of the lattice with its first neighbors. By applying an external magnetic field, the Hamiltonian of the system is then

$$
H_{o}=-\frac{J}{2} \sum_{<i, j>a, b=1} \sum_{a b}^{3} h_{i}^{a} S_{j}^{b}-\mathrm{g} \mu \sum_{i} \sum_{a=1}^{3} S_{i}^{a} B_{a}
$$

Here $h_{a b}$ is the $3 \times 3$ dimensionless interaction law matrix (metric of the internal space of spins), $J$ is the exchange energy, and $S_{i}^{a}$ denotes the $a$-th component of the spin of the $i$-th cell of the lattice, while $\langle i, j\rangle$ includes in the sum only the first neighbor cells $j$ of each cell $i$. For isotropic interaction we have $h_{a b}=\operatorname{diag}(1,1,1)$. The parameter $\mathrm{g}$ is the gyromagnetic factor of the spins in the material medium, $\mu$ is their magnetic momentum and $B_{a}$ is the $a$-th component of the external magnetic field. Eq. (1) is the hamiltonian for $J>0$, describing the ferromagnetic case. In the antiferromagnetic case $(J<0)$, the spin vector $\vec{S}$ must be replaced by the Néel vector $\vec{\eta}=\frac{1}{2}\left(\vec{S}_{1}-\vec{S}_{2}\right)$, where the index distinguish two distinct sub-lattices in the more simple case.

For sufficiently small distances between neighbor cells (and large spins) we can pass to the continuous limit, retaining only up to second order terms in the Taylor expansion in this parameter. In this limit, the hamiltonian for a spin lattice with a non-trivial geometry is then given by the nonlinear sigma model as

$$
H=2 J \iint_{S} \sqrt{|g|} d x^{1} d x^{2}\left[\frac{1}{4} \sum_{a, b=1}^{3} \sum_{\alpha, \beta=1}^{2} h_{a b} g^{\alpha \beta} \frac{\partial S^{a}}{\partial x^{\alpha}} \frac{\partial S^{b}}{\partial x^{\beta}}-\frac{1}{2 \rho_{B}^{2}} \sum_{a=1}^{3} \frac{S^{a} B_{a}}{B}\right]
$$

Here $g_{\alpha \beta}$ is the metric of the surface and $g^{\alpha \beta}$ is its inverse, $\sqrt{|g|} d x^{1} d x^{2}$ is the infinitesimal area element, $x^{1}$ and $x^{2}$ are coordinates which describe the surface, $g=\operatorname{det}\left[g_{\alpha \beta}\right], \rho_{B}^{2}=$ $\frac{2 J}{\mathrm{~g} \mu B}$ and $B$ is the magnitude of the external magnetic field.

In our case, the spins lie on an infinite cylinder of radius $\rho_{o}$, with deficit angle $2 \pi(1-c)$ and screw dislocation $k$ [1215]. This 2-dimensional manifold is characterized by the line element written in cylindrical coordinates as

$$
d s^{2}=\left(c^{2} \rho_{o}^{2}+k^{2}\right) d \varphi^{2}+2 k d \varphi d z+d z^{2} .
$$

If $c=1$ and $k=0$ then clearly it includes the case of a typical cylinder. In order to achieve the geometry (3), we can remove an angular sector and make a longitudinal dislocation from the flat surface (with metric given by $d s^{2}=\rho_{o}^{2} d \theta^{2}+d Z^{2}$ ), and then identify the points at the edges as $\left(\rho_{o}, \theta, Z\right) \leftrightarrow\left(\rho_{o}, \theta+2 \pi c, Z+2 \pi k\right)$. With new coordinates 
$\varphi \equiv \theta / c \quad$ and $\quad z \equiv Z-(k / c) \theta$, the line element takes the form of Eq. (3) while the usual identification $\left(\rho_{o}, \varphi, z\right) \leftrightarrow$ $\left(\rho_{o}, \varphi+2 \pi, z\right)$ must be observed.

The equations of the model can be obtained by writing the vector $\vec{S}=(\sin \Theta \cos \Phi, \sin \Theta \cos \Phi, \cos \Theta)$ in spherical coordinates. By applying the principle of least action for the hamiltonian (2) on the geometry (3) and by making $B_{a}=(0,0, \pm B)$ we obtain two highly nonlinear partial differential equations. We are searching symmetric solutions, the generalization of cylindrically symmetric ones in the usual case (say with $c=1$ and $k=0$ ). Therefore, we make the supposition $\Phi(\varphi, z)=\varphi$ to yield

$$
\frac{\partial^{2} \Theta}{\partial z^{2}}=\alpha \sin \Theta+\beta \sin 2 \Theta
$$

$\mathrm{e}$

$$
\frac{\partial \Theta}{\partial \varphi}=k \frac{\partial \Theta}{\partial z}
$$

respectively. These are the Double Sine-Gordon (DSG) and helical equations, where $\alpha= \pm \frac{1}{\rho_{B}^{2}}$ and $\beta=\frac{1}{2 c^{2} \rho_{o}^{2}}$.
There is a large number of solutions to the DSG (4). However, we need to seek new solutions in order to comply with the boundary conditions $\Theta(\varphi, z) \equiv \Theta(\varphi+2 \pi, z)$ and $\Phi(\varphi, z) \equiv \Phi(\varphi+2 \pi, z)$. These boundary conditions are necessary in order to have single-valued spin vector and also to satisfy the helical equation (5). Some methods have been proposed in order to solve nonlinear differential equations. For instance, the homogeneous balancement method [16], the method of expansion in hyperbolic functions [17-19], the method of test function [20,21], the method of nonlinear transformation [22, 23], and sine-cosine method [24]. However, these methods yield only the solutions of solitary waves and shock waves. These methods do not yield periodic solutions of nonlinear equations. Although the method of expansion in Jacobi elliptic functions includes periodic solutions, we use here a more general method for solving our system of equations $[25,26]$. This method has been known as the $\mathrm{f}-$ expansion method [27, 28]. A few stable solutions have been found in terms of the Jacobi elliptic functions [29]. A subset of them reads as follows

$$
\begin{aligned}
\Theta(\varphi, z)= & 2 \arctan \left\{\sqrt{\frac{2-m^{2}}{2\left(1-m^{2}\right)}-\frac{(\alpha+2 \beta) m^{4}}{2\left(1-m^{2}\right)\left(2 \beta\left(2-m^{2}\right)+\sqrt{4 \beta^{2}\left(2-m^{2}\right)^{2}+\left(\alpha^{2}-4 \beta^{2}\right) m^{4}}\right)}}\right. \\
& \left.\times \operatorname{cs}\left[\sqrt{\frac{2 \beta\left(2-m^{2}\right)+\sqrt{4 \beta^{2}\left(2-m^{2}\right)^{2}+\left(\alpha^{2}-4 \beta^{2}\right) m^{4}}}{m^{4}}}(z+k \varphi)\right]\right\} .
\end{aligned}
$$

Here $m \in(0,1)$ is the module of the Jacobi elliptic function cs. The function cs is the ratio of sn and $\mathrm{cn}$, which respectively are the Jacobi elliptic sine and cosine functions [31]. The value for $m$ is found by equaling the periodicity of the Jacobi elliptic functions $\Omega(m)=4 \int_{0}^{1} \frac{d t}{\sqrt{\left(1-t^{2}\right)\left(1-m^{2} t^{2}\right)}}$, which is itself an elliptic function of first kind, with the periodicity required by the boundary conditions $T(m)=$ $2 \pi k\left\{\sqrt{\frac{2 \beta\left(2-m^{2}\right)+\sqrt{4 \beta^{2}\left(2-m^{2}\right)^{2}+\left(\alpha^{2}-4 \beta^{2}\right) m^{4}}}{m^{4}}}\right\}$. In Fig. 1 we depict schematically the spin field on the cylinder according to the solution (6) due only to the interactions among spins with $\alpha=0$.

For the usual cylinder the equilibrium configuration with the lowest energy for the first class of homotopy is a $\pi$-soliton along the whole cylinder [30]. In our case, we see clearly a multi- $\pi$-soliton structure. Cylindrical symmetry is shown there while we have a helical configuration around the cylinder here. This difference is associated to the lattice structure which was longitudinally dislocated.

We discuss now the main effects due to the screw dislocation and the deficit angle (relative to a simple cylinder). The screw dislocation $k$ eliminates the state of single solitons, typical of a cylinder without this defect, allowing only

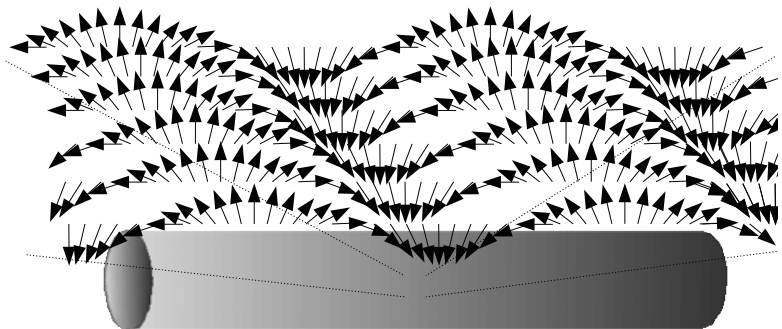

FIG. 1: Schematic representation of the spin distribution on the cylinder according to solutions (6). We depicted the solution for the case without magnetic field considering only the interactions among spins. The cylinder and the spin periodic structure are presented in different scales. The spin configuration should be seen as the amplification from a small flat region of the cylinder. There is a dislocated spin configuration, which is a helical configuration along the whole cylinder.

periodic states. It introduces periodic boundary conditions in order to make the spin vector single-valued. These boundary conditions must be satisfied for all solutions. Therefore, the solutions need also to be periodic. This is a property of this 
defect. In all other base manifold which has solutions with axial symmetry, a screw dislocation could similarly eliminate the state of a single soliton from the system. On the other hand, the deficit angle introduces a possibility to scale the radius of the cylinder in such a way that a simple cylinder and a cylinder with conicity may have the same magnetic properties due to the spin textures. This is shown clearly in Eq. (3). The parameter $c$ of conicity is a multiplicative factor of the radius $\rho_{o}$ of the cylinder.

The graphics from Figs. 2 and 3 illustrate the behavior of the system as function of the magnetic field for two opposite directions along the whole cylinder.

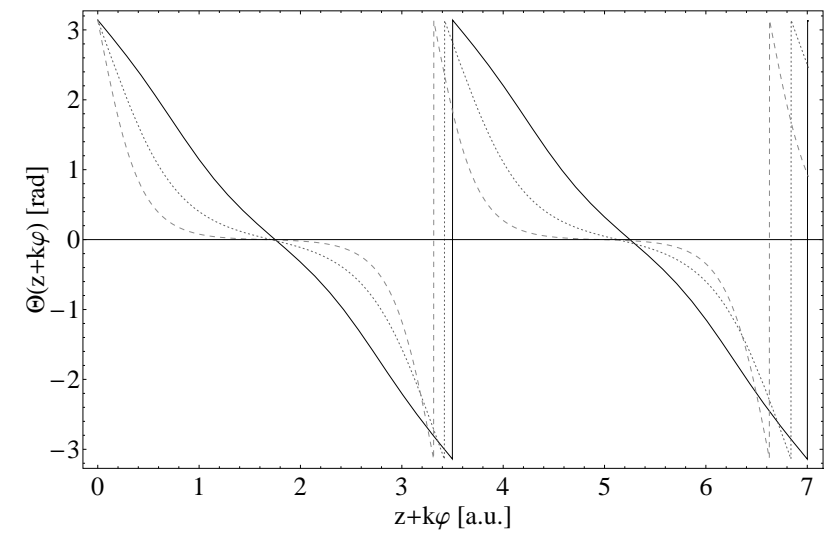

FIG. 2: Solid, dotted and dashed lines represent the qualitative behavior of the function $\Theta$ from Eq. (6) for $\alpha=0,4,14$ with $\beta=1$ and $k=1$ in the range $0 \leq z+k \varphi \leq 7$, respectively. It is clear the displacement of the domain-walls and the appearance of a saturated region by increasing the magnetic field.

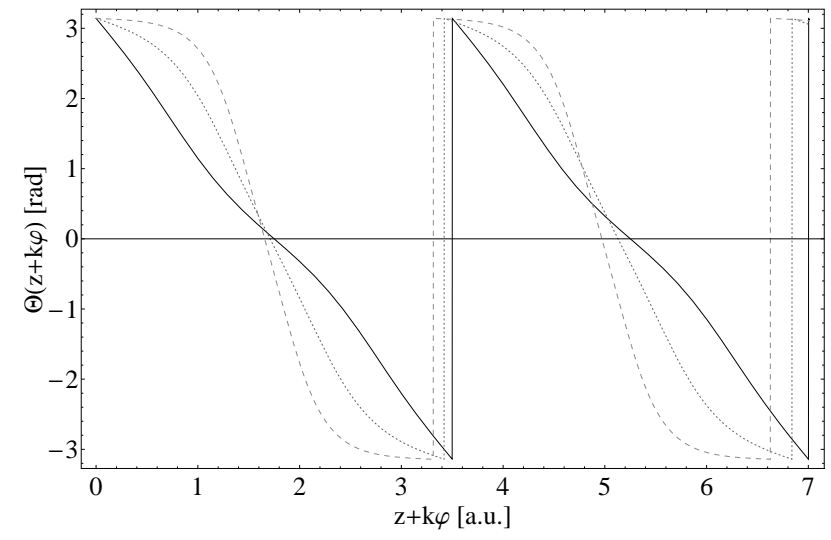

FIG. 3: The same as Fig. 2 for $\alpha=0,-4,-14$.

We see that the spin vector periodically assumes all values from $-\pi$ to $\pi$ while $z+k \varphi$ grows. In each period there is a confined $2 \pi$-soliton, characterizing a multi-solitonic state along the whole cylinder. By increasing the magnetic field we dislocate the spin vectors in such a way that we may configure two different regions. A saturation region with $\Theta=0[\pi]$ and a region with $\Theta$ changing in the range $(-\pi, \pi)$. We can associate the saturation region to the magnetic domains separated by domain-walls, which are the $2 \pi$-solitons occurring in the region where $\Theta$ varies.

Since the solution (6) satisfies both the DSG (4) and the helical equation (5), the exchange energy density is given by

$$
\mathcal{H}=J \Theta_{z}^{2}
$$

Here $\Theta_{z}$ is the partial derivative of the solution (6) with respect to the variable $z$. From Eq. (7), Figs. 2 and 3 we see that the magnetic energy density concentrates in the region of the domain-walls while the magnetic field increases. The function $\Theta_{z}$ increases rapidly in this region and vanishes in the saturated region.

The magnitude of the magnetic field does not need to be homogeneous along the whole cylinder. Therefore, we may apply, starting from a configuration without magnetic field with $\alpha=0$, a local magnetic field into a region and reverse the local magnetic field into another region. Then we solve Eqs. (4) and (5) for this configuration of different magnetic fields in order to obtain the same solution form (6) in these regions but with different magnetic field. This local magnetic field may be a mechanism for the magnetic recording since it can produce two distinct states with $\Theta=0[\pi]$ isolated by $\pi$-soliton-like domain-walls.

According to solution (6) for each step $2 \pi k$ the $\Theta$ component of the spin vector undertakes a rotation of $2 \pi \mathrm{rad}$, at least once, to fulfill the boundary conditions. After performing a full $2 \pi$ rad turn around the cylinder, covering $2 \pi \rho_{o}$, the solution also undertakes this full rotation. For a nanotube we can make $\rho_{o} \approx 10^{-9} m, k \approx 10^{-9} m$ and $c=1[32,33]$. If each domain is a byte, then we have at least one byte in the area $4 \pi^{2} \rho_{o} k \approx 10^{-13} i n^{2}$. Thus the density of magnetic recording may reach beyond the order of Terabytes $/ \mathrm{in}^{2}$, which is much larger than the current capacity of about Gigabytes $/ \mathrm{in}^{2}$. The smaller the cylinder's radius and the dislocation, the greater will be the density of bytes.

Our periodic solutions with magnetic field does not fulfill the self-dual equations of Bogomol'nyi, obtained from our hamiltonian and given by

$$
\begin{aligned}
\frac{\partial \Theta}{\partial z} & = \pm \beta \sin \Theta\left(\frac{\partial \Phi}{\partial \varphi}-k \frac{\partial \Phi}{\partial z}\right), \\
\beta\left(\frac{\partial \Theta}{\partial \varphi}-k \frac{\partial \Theta}{\partial z}\right) & =\mp \sin \Theta \frac{\partial \Phi}{\partial z}
\end{aligned}
$$

This would be necessary in order to minimize the energy in each class of homotopy to $8 \pi J|Q|$, where $Q$ is the topological charge on a finite cylindrical section, causing geometrical frustration. This feature is due to the periodic solution caused by the screw-dislocation and the presence of the magnetic length $\rho_{B}$. Since these equations are not fulfilled, the energy $H(>8 \pi J|Q|)$ in this cylindrical section may be minimized by the deformation of an elastic cylinder whose constraint $\rho(z, \varphi)=\rho_{o}$ may be removed along the whole cylinder. The regions where the domain-walls are localized are deformed perpendicularly to the axis of the elastic cylinder. It is clear that these deformations may also change the solutions in order to eliminate the geometrical frustration [34].

From Figs. 2 and 3 it can be concluded that the homogeneous variation of the magnetic field along the whole 
cylinder (not local) dislocates synchronized with the domainwalls. This global variation of the magnetic field may produce a spatial movement of these deformations causing a peristaltic state [35]. This movement could be useful for the transport of molecules or nano-structures and also for the production of mechanic energy.

The known case $k=0$ of spins in a nondislocated cylinder cannot be found from the limit of our solution (6) as $k \rightarrow 0$. This solution diverges when trying to obtain a single-soliton. This would occur if it were a hyperbolic function $(m \rightarrow 1)$. If the parameter $k$ of screw dislocation vanishes, then our solution would no longer depend of the variable $\varphi$. The periodic boundary conditions would then be naturally satisfied, even if the solution were not periodic. The feature of this work is that soliton-like localized solutions naturally occur for $k=0$, but are absent for $k \neq 0$ as states with the lowest energy.

It is known in the science of materials how discordances (screw dislocation) alter properties like hardness. We have seen here how this defect also changes significantly its mag- netic properties. In particular, discordances would be related to the formation of magnetic domain and effects of geometrical frustration. This work may be of particular interest in nanotechnology. Only recently began the synthesis of nanotubes and nanowires. On nanotubes and nanowires it may be possible the deposition of ferromagnetic mono-coatings with screw dislocation. The spin textures discussed here could play an important role in technological applications such as the construction of magnetic recording devices. They may also be useful in order to understand phenomena in biological systems, if organic microtubules with some kind of ferromagnetic magnetization can be found in nature. The method utilized here can also be important for others physical problems.

We are grateful to R. Klippert, V. De Lorenci, Winder A. Moura-Melo, V. Pershin and A. K. T. Assis for their criticisms and suggestions. We also thank FAPEMIG and CAPES for financial support and Itajubá Federal University by the hospitality.
[1] E. A. Silva and A. R. Pereira, Phys. Status Solidi B 213, 481 (1999).

[2] E. A. Silva and A. R. Pereira, Solid State Commun. 113, 669 (2000).

[3] V. L. Carvalho-Santos, A. R. Moura, W. A. Moura-Melo and A. R. Pereira, Phys. Rev. B 77, 134450 (2008)

[4] L. R. A. Belo, N. M. Oliveira-Neto, W. A. Moura-Melo, A. R. Pereira and E. Ercolessi, Phys. Lett. A 365, 463 (2007).

[5] R. Dandoloff and A. Saxena, Eur. Phys. J. B 29, 265 (2002).

[6] A. Saxena and R. Dandoloff, Phys. Rev. B 66, 104414 (2002).

[7] A. Saxena and R. Dandoloff, Phys. Rev. B 55, 11049 (1997).

[8] A. Saxena, R. Dandoloff and T. Lookman, Physica A 261, 13 (1998).

[9] C. Itzykson and J. B. Zuber, Quantum Field Theory (McGRAW-HILL, New York, 1980), ed. 1.

[10] T. Uhlig, M. Rahm, C. Dietrich, R. Hollinger, M. Heumann, D. Wiess and J. Zweck, Phys. Rev. Lett. 95, 237205 (2005).

[11] M. Rahm, J. Stahl, W. Wegscheider and D. Wiess, Appl. Phys. Lett. 85, 1553 (2004).

[12] V. A. De Lorenci and E. S. Moreira Jr., Phys. Rev. D 67, 124002 (2003)

[13] A. Saxena and R. Dandoloff, Phys. Rev. B 66, 104414 (2002).

[14] A. R. Pereira, J. M. M. M. 285, 60 (2005).

[15] Moura-Melo et al., Phys. Lett. A 360, 472 (2007).

[16] M.L. Wang, Phys. Lett A 199, 169 (1995)

[17] L. Yang, J. Liu and K. Yang, Phys. Lett. A 278, 267 (2001).

[18] B.R. Duffy, Phys. Lett. A 214, 271 (1996).

[19] E.J. Parkes, Z. Zhu, B.R. Duffy and H.C. Huang, Phys. Lett.
A 248, 219 (1998).

[20] R. Hirota, J. Math. Phys. 14, 810 (1973).

[21] N.A. Kudryashov, Phys. Lett. A 147, 287 (1990).

[22] M. Otwinowski, R. Paul and W.G. Laidlaw, Phys. Lett. A 128, 483 (1988)

[23] S.K. Liu, Z.T. Fu, S.D. Liu and Q. Zhao, Appl. Math. Mech. 22, 326 (2001).

[24] C. Yan, Phys. Lett A 224, 77 (1996).

[25] S.K. Liu, Z.T. Fu, S.D Liu and Q. Zhao, Phys. Lett. A 289, 69 (2001).

[26] Z.T. Fu, S.K. Liu, S.D. Liu and Q. Zhao, Phys. Lett. A 290, 72 (2001).

[27] Y.Z. Peng, Phys. Lett. A 314, 401 (2003).

[28] E. Yomba, Phys. Lett. A 340, 149 (2005).

[29] L. A. N. de Paula, Master's thesis, Itajubá Federal University, 2008.

[30] A. Saxena and R. Dandoloff, Phys. Rev. B 58, R563 (1998).

[31] V. Prasolov, Y. Solovyev, Elliptic Functions and Elliptic Integrals (American Mathematical Society, Providence, 1997).

[32] The Nonotube Site, available from http://www.pa.msu.edu/cmp/csc/nanotube.html.

[33] A. C. M. Carvalho, PhD thesis, Campinas State University, 2004.

[34] S. Villain-Guillot, R. Dandoloff, A. Saxena and A. R. Bishop, Phys. Rev. B 52, 6712 (1995).

[35] R. Bar-Ziv and E. Moses, Phys. Rev. Lett. 73, 1392 (1994). 